\title{
Single-institutional outcome-analysis of low-dose stereotactic body radiation therapy (SBRT) of adrenal gland metastases
}

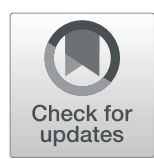

Theresa Voglhuber $^{1 *}$ (D), Kerstin A. Kessel ${ }^{1,2,3}$, Markus Oechsner ${ }^{1}$, Marco M. E. Vogel ${ }^{1}$, Jürgen E. Gschwend ${ }^{4}$ and Stephanie E. Combs ${ }^{1,2,3}$

\begin{abstract}
Background: Adrenal gland metastases are a common diagnostic finding in various tumor diseases. Due to the increased use of imaging methods, they are diagnosed more frequently, especially in asymptomatic patients. SBRT has emerged as a new, alternative treatment option in the field of radiation oncology. In the past, it was often used for treating inoperable lung, liver, prostate, and brain tumors. Meanwhile, it is also an established keystone in the treatment of oligometastatic diseases. This retrospective study aims to evaluate the effect of low-dose SBRT in patients with adrenal metastases.
\end{abstract}

Methods: We analyzed a group of 31 patients with 34 adrenal gland lesions treated with low-dose SBRT between July 2006 and July 2019. Treatment-planning was performed through contrast-enhanced CT, followed by imageguided stereotactic radiotherapy using cone-beam CT. The applied cumulative median dose was $35 \mathrm{~Gy}$; the median single dose was $7 \mathrm{~Gy}$. We focused on local control (LC), progression-free survival (PFS), overall survival (OS), as well as acute and late toxicity.

Results: Seven adrenal gland metastases (20.6\%) experienced local failure, $80.6 \%$ of the patients faced a distant progression. Fourteen patients were still alive. Median follow-up for all patients was 9.8 months and for patients alive 14.4 months. No treatment-related side-effects > grade 2 occurred. Of all, $48.4 \%$ suffered from acute gastrointestinal disorders; $32.3 \%$ reported acute fatigue, throbbing pain in the renal area, and mild adrenal insufficiency. Altogether, 19.4\% of the patients faced late-toxicities, which were as follows: Grade 1: 12.9\% gastrointestinal disorders, 3.2\% fatigue, Grade 2: 9.7\% fatigue, 6.5\% headache, 3.2\% loss of weight. The 1-year OS and probability of LF were 64 and $25.9 \%$, respectively.

Conclusion: Low-dose SBRT has proven as an effective and safe method with promising outcomes for treating adrenal metastases. There appeared no high-grade toxicities $>$ grade 2 , and $79.4 \%$ of treated metastases were progression-free. Thus, SBRT should be considered as a therapy option for adrenal metastases as an individual therapeutic concept in the interdisciplinary discussion as an alternative to surgical or systemic treatment.

Keywords: Stereotactic body radiation therapy, Adrenal gland metastases, Oncology, Outcome, SBRT, Toxicity, Survival

\footnotetext{
* Correspondence: voglhuber@live.at

'Department of Radiation Oncology, Technical University of Munich (TUM), Ismaninger Straße 22, Munich, Germany

Full list of author information is available at the end of the article
}

(c) The Author(s). 2020 Open Access This article is licensed under a Creative Commons Attribution 4.0 International License, which permits use, sharing, adaptation, distribution and reproduction in any medium or format, as long as you give appropriate credit to the original author(s) and the source, provide a link to the Creative Commons licence, and indicate if changes were made. The images or other third party material in this article are included in the article's Creative Commons licence, unless indicated otherwise in a credit line to the material. If material is not included in the article's Creative Commons licence and your intended use is not permitted by statutory regulation or exceeds the permitted use, you will need to obtain permission directly from the copyright holder. To view a copy of this licence, visit http://creativecommons.org/licenses/by/4.0/. The Creative Commons Public Domain Dedication waiver (http://creativecommons.org/publicdomain/zero/1.0/) applies to the data made available in this article, unless otherwise stated in a credit line to the data. 


\section{Background}

The adrenal gland is a frequent target of malignant tumor cells of different entities, as it is characterized by a rich blood supply [1]. To make the right therapy decision, it is essential to differentiate between various benign and malignant neoplasia of the tissue. For example, pheochromocytomas arise from the cells of the adrenal medulla, while benign adenomas and metastatic lesions mainly form in the cortical regions [2]. Therefore, radiologic imaging is an indispensable tool for distinguishing between the most diverse types of tumors, as these have different characteristics on computed tomography (CT) and magnet resonance imaging (MRI) [3]. The diagnostic proceeding of adrenal metastases has undergone significant changes lately. In the past, lesions of the adrenal gland were incidental findings, manifested by symptomatic development, or were detected at autopsies [4, 5]. Since the introduction of systematic and more accurate tumor staging and the high-frequency use of imaging, the incidence of adrenal tumors has increased, as they are frequently discovered by chance, without any suggestive symptomatology $[4,6]$.

Once the adrenal gland metastasis is diagnosed through imaging procedures or biopsy, there are several options for curative and palliative treatment. Particularly for patients with isolated lesions, laparoscopic or open surgery is performed. Further treatment options are systemic chemo-/immunotherapy or ablative strategies such as cryoablation and radiofrequency ablation [3]. In the past, hypofractionated radiotherapy (RT) was often used for palliative purposes to reduce physical complaints caused by symptomatic adrenal metastases, such as back/flank pain, nausea, or hypoadrenalism [7]. With the introduction of stereotactic body radiation therapy (SBRT), a promising option for treating adrenal gland metastases appeared in the field of radiation oncology. Previously SBRT has been very extensively used in treating inoperable lung, liver, prostate, and brain tumors [8].

SBRT is characterized by its excellent local tumor control and is a good, non-invasive alternative treatment option, especially in patients, who decline any invasive- or ablative treatment, or when surgery is contraindicated due to multiple comorbidities $[3,6$, 9-14]. The high-precision irradiation is applied in high single doses and few fractions. Due to the rapid dose-reduction outside the target volume, surrounding, healthy structures can be spared $[6,8,15,16]$. This present retrospective study aims to contribute to the knowledge regarding hypofractionated low-dose SBRT of adrenal gland metastases. In particular, the primary endpoints local control (LC), progression-free survival (PFS) and overall survival (OS) will be evaluated as well as acute and late toxicity rates.

\section{Methods}

\section{Patients}

A group of 31 patients with a total of 34 irradiated lesions (3 patients had metastases treated on both adrenal glands) were evaluated. All were treated at the department of radiation oncology, Klinikum rechts der Isar, Technical University of Munich (TUM) between July 2006 and July 2019. Table 1 shows patient characteristics. The inclusion criterion was RT treatment with low-dose SBRT due to adrenal metastases. A progressive disease, uncontrolled primary and multifocal tumor manifestation were allowed. The guidelines of the german DEGRO (Deutsche Gesellschaft für Radioonkologie) working group for stereotactic RT (AG Stereotaxie) were applied to define SBRT. All patients were primarily treated in a palliative setting, for example, due to an oligoprogression in the area of the adrenal gland with otherwise stable tumor disease, to prolong survival, or to treat tumor dependent symptoms. An individual curative approach was attempted in three of the patients with oligometastases.

Analysis of patient records and data collection took place after being approved by the local ethics committee of the Medical faculty of TUM, vote number 307/19.

\section{Treatment}

Treatment planning was performed through contrastenhanced CT. Five patients received an additional MRI and four patients an additional positron emission tomography-CT (PET-CT) for evaluating the tumor volume. Since 2010, 4D-CT was also acquired. Time-resolved imaging allows more accurate reconstruction of respiratory-related tumor movements, which also helps to protect surrounding healthy tissue $[17,18]$. Before the $4 \mathrm{D}$ CT was introduced, positioning the patient through frames, blue bag, abdominal press, wing board, and breathing techniques ensured motion management. Before irradiation, the patient's exact location was controlled by using a conebeam CT (CBCT) to ensure high-precision. During the planning simulation and during irradiation itself, patients were brought in a supine position and immobilized with the help of a vacuum mattress, a wingstep, and a knee wedge to further reduce irradiated uncertainties.

The median applied cumulative dose was $35 \mathrm{~Gy}$ (range: 25-57 Gy), and the median single dose was 7 Gy (range: 3$8 \mathrm{~Gy}$ ) in 5 fractions (range: $3-14$ ). This results in a median biological equivalent dose (BED) of $59.5 \mathrm{~Gy}$ (range: $37.5-$ $72.0 \mathrm{~Gy}$ ). The calculated doses were prescribed to the 60 $80 \%$ isodose. Two patients received boost treatment with a total dose of 42 Gy (35 á 2.5 Gy base plan with a simultaneous integrated boost of 42 á 3 Gy) and 57 Gy (45 á 1.8 Gy base plan with a sequential boost of 12 á $4 \mathrm{~Gy}$ ).

The treatment concept was determined in an interdisciplinary discussion, depending on tumor size, symptoms, and general condition of the patients. 
Table 1 Patient characteristics

\begin{tabular}{|c|c|}
\hline Characteristics & Values \\
\hline Number of patients (n) & 31 \\
\hline Number of AGMs (n) & 34 \\
\hline \multicolumn{2}{|l|}{ Gender } \\
\hline Male & $17(54.8 \%)$ \\
\hline Female & $14(45.2 \%)$ \\
\hline Age at SBRT (median, range) [years] & $66.1(26.7-82.2$ \\
\hline \multicolumn{2}{|l|}{ Primary entities } \\
\hline NSCLC & $13(41.9 \%)$ \\
\hline Mamma Ca & $6(19.4 \%)$ \\
\hline Melanoma & $4(12.9 \%)$ \\
\hline Others & $8(25.8 \%)$ \\
\hline \multicolumn{2}{|l|}{ Symptoms } \\
\hline Present & $2(6.5 \%)$ \\
\hline Absent & 29 (93.5\%) \\
\hline \multicolumn{2}{|l|}{ KPS } \\
\hline $100 \%$ & $1(3.2 \%)$ \\
\hline $90 \%$ & $19(61.3 \%)$ \\
\hline $80 \%$ & $10(32.3 \%)$ \\
\hline$\leq 70 \%$ & $4(12.9 \%)$ \\
\hline \multicolumn{2}{|l|}{ Location of AGMs } \\
\hline Left & 15 (48.4\%) \\
\hline Right & $13(41.9 \%)$ \\
\hline Bilateral & $3(9.7 \%)$ \\
\hline \multicolumn{2}{|l|}{ Controlled primary } \\
\hline Yes & $25(80.6 \%)$ \\
\hline No & $6(19.4 \%)$ \\
\hline \multicolumn{2}{|l|}{ AGM diagnosis } \\
\hline Synchronous & 15 (48.4\%) \\
\hline Metachronous & $19(61.3 \%)$ \\
\hline \multicolumn{2}{|l|}{ Metastases in other sites } \\
\hline Yes & $25(80.6 \%)$ \\
\hline No & $6(19.4 \%)$ \\
\hline \multicolumn{2}{|c|}{ Systemic therapy within four weeks before/after SBRT } \\
\hline Yes & $19(61.3 \%)$ \\
\hline No & $12(38.7 \%)$ \\
\hline FU-time (median, range) [months] & $9.8(0-120.5)$ \\
\hline
\end{tabular}

AGM adrenal gland metastasis, SBRT stereotactic body radiation therapy, NSCLC non-small-cell-lung-cancer, KPS Karnofsky Performing Score, FU follow-up synchronous $\leq 3$ months after initial primary diagnosis; metachronous $>3$ months after initial primary diagnosis

\section{Follow-up}

The first regular follow-up (FU) appointment was arranged 4-6 weeks after SBRT. Each additional exam took place approximately every three months in the first year and then every 6-12 months. Depending on tumor progression or worsening of symptoms, follow-up appointments could be individualized. Each appointment included a full physical exam, a consultation with a radiation specialist, and contrast-enhanced CT to assess the tumor status and therapy-associated side-effects. LC of the adrenal metastasis was assessed in each follow-up CT, regardless of the primary tumor status.

Every patient was examined for side-effects, such as fatigue, nausea, or abdominal pain, during and after treatment. We determined these side-effects according to the Common Terminology Criteria Adverse Events (CTCAE) Version 5.0. Due to the retrospective design of this study, the level of toxicity could not be precisely assessed in some cases; grading according to CTCAE was then carried out using documented follow-up reports, written by the supervising radio-oncologist and the development of the patient's clinical course. We defined side-effects as either acute when they appeared within the first six months after RT or as late when they appeared after the six-monthsperiod.

\section{Statistics}

Based on the prevailing competing risks (e.g., death of a patient before a local failure occurred), the probability of LF was calculated using a competing-risk-analysis in order to increase accuracy $[19,20]$. It was performed with R-Statistics (R-Foundation, Vienna). We used SPSS Statistics v25 (IBM, USA) for all other analyses.

For patients with multiple RT on both adrenal glands, the first treatment was used for the calculation of OS and PFS; for LC, all 34 cases were included. We calculated the LC from the last day of irradiation until local failure of the treated lesion or last day of follow-up. PFS was calculated from the last day of irradiation until general tumor progression or last day of follow-up; OS from the last day of irradiation until the date of death or the last date the patient was alive. Receiver operating characteristics (ROC) were used to determine thresholds for grouped variables. Cox regression was used for the analysis of subgroups. We considered a $p$-value of $<0.05$ as significant.

The $\mathrm{BED}_{10}$ was calculated with the formula; $\mathrm{BED}(\mathrm{Gy})=$ dose/fraction $x$ fraction number $(1+$ fraction dose $/ \alpha / \beta)$; we used an $\alpha / \beta$ ratio of 10 for the tumor tissue, see Table 2 [21].

\section{Results}

The median age was 66 years (range: $27-82$ years). Of all, $25(25 / 31,81 \%)$ patients suffered from advanced tumor disease with multiple metastases in different organs. 28 $(28 / 31,90 \%)$ patients showed an oligoprogression with a maximum of $\leq 3$ metastases/tumor areas. In almost all patients $(25 / 31,81 \%)$, the primary tumor was controlled before irradiation. Isolated adrenal metastases without further tumor manifestation occurred in six $(6 / 31,19 \%)$ patients. Only two $(2 / 31,6 \%)$ patients reported symptoms 
Table 2 Radiation parameters and treatment characteristics

\begin{tabular}{|c|c|c|c|c|c|c|c|c|c|c|c|c|}
\hline ID & PTV (ml) & GTV (ml) & TD (Gy) & SD (Gy) & Fractions & Isodose & PTV-Dmax (Gy) & PTV-D2\% (Gy) & PTV-D50\% (Gy) & $\begin{array}{l}\text { PTV-D98\% } \\
\text { (Gy) }\end{array}$ & GTV-D50\% (Gy) & $\mathrm{BED}_{10}$ \\
\hline 1 & 175.8 & 57.8 & 25.0 & 5.0 & 5.0 & $60 \%$ & 42.8 & 42.3 & 37.7 & 29.0 & 41.3 & 37.5 \\
\hline 2 & 376.0 & 225.5 & 25.0 & 5.0 & 5.0 & $60 \%$ & 28.1 & 27.8 & 26.6 & 24.6 & 26.8 & 37.5 \\
\hline 3 & 123.0 & 53.8 & 25.0 & 5.0 & 5.0 & $60 \%$ & 41.8 & 41.7 & 39.6 & 29.7 & 41.1 & 37.5 \\
\hline 4 & 45.0 & $x$ & 25.0 & 5.0 & 5.0 & $60 \%$ & $x$ & 25.3 & 21.0 & $x$ & $x$ & 37.5 \\
\hline 5 & 39.3 & 14.0 & 25.0 & 5.0 & 5.0 & $60 \%$ & 42.0 & 41.5 & 34.5 & 26.3 & 39.1 & 37.5 \\
\hline 6 & 37.3 & 8.3 & 35.0 & 7.0 & 5.0 & $65 \%$ & 54.3 & 53.8 & 47.3 & 33.7 & 52.6 & 59.5 \\
\hline $7 a$ & 40.7 & 12.9 & 40.0 & 8.0 & 5.0 & $65 \%$ & 61.7 & 59.2 & 48.8 & 39.0 & 53.8 & 72.0 \\
\hline $7 b$ & 24.1 & 6.9 & 40.0 & 8.0 & 5.0 & $65 \%$ & 61.0 & 59.7 & 49.1 & 40.5 & 55.3 & 72.0 \\
\hline 8 & 87.4 & 13.3 & 35.0 & 7.0 & 5.0 & $60 \%$ & 47.1 & 46.3 & 40.4 & 22.8 & 44.5 & 59.5 \\
\hline 9 & 71.9 & 21.0 & 25.0 & 5.0 & 5.0 & $60 \%$ & 43.1 & 30.5 & 23.9 & 30.6 & 41.5 & 37.5 \\
\hline 10 & 98.8 & 37.3 & 25.0 & 5.0 & 5.0 & $60 \%$ & $x$ & 25.5 & 23.3 & $x$ & 24.6 & 37.5 \\
\hline 11 & 69.8 & 25.6 & 40.0 & 8.0 & 5.0 & $65 \%$ & 61.9 & 60.3 & 49.3 & 39.9 & 54.8 & 72.0 \\
\hline 12 & 134.0 & 55.0 & 35.0 & 7.0 & 5.0 & $60 \%$ & $x$ & 37.7 & 31.9 & $x$ & 34.0 & 59.5 \\
\hline 13 & 122.2 & 51.3 & 35.0 & 7.0 & 5.0 & $60 \%$ & 59.9 & 58.8 & 44.3 & 22.1 & 51.5 & 59.5 \\
\hline 14 & 49.2 & 12.7 & 25.0 & 5.0 & 5.0 & $60 \%$ & 41.9 & 41.9 & 38.6 & 30.1 & 41.0 & 37.5 \\
\hline 15 & 82.8 & 33.0 & 25.0 & 5.0 & 5.0 & $80 \%$ & 31.7 & 31.5 & 29.8 & 25.1 & 30.9 & 37.5 \\
\hline 16 & 294.2 & 154.6 & 36.0 & 3.0 & 12.0 & $60 \%$ & 62.7 & 59.2 & 47.6 & 33.3 & 50.6 & 46.8 \\
\hline 17 & 434.8 & 247.5 & 27.0 & 5.4 & 5.0 & $60 \%$ & 45.9 & 44.6 & 37.1 & 27.8 & 40.9 & 41.6 \\
\hline 18 & 35.8 & 3.1 & 35.0 & 7.0 & 5.0 & $60 \%$ & 61.8 & 60.8 & 47.6 & 31.9 & 58.9 & 59.5 \\
\hline 19 & 81.6 & 22.3 & 35.0 & 7.0 & 5.0 & $60 \%$ & 63.3 & 62.7 & 49.3 & 28.4 & 60.5 & 59.5 \\
\hline 20 & 138.7 & 71.4 & 25.0 & 5.0 & 5.0 & $60 \%$ & 39.5 & 37.7 & 26.3 & 25.3 & 27.6 & 37.5 \\
\hline 21 & 58.5 & 12.2 & 35.0 & 7.0 & 5.0 & $60 \%$ & 58.6 & 58.3 & 54.3 & 41.4 & 57.7 & 59.5 \\
\hline 22 & 37.9 & 7.7 & 35.0 & 7.0 & 5.0 & $65 \%$ & 56.0 & 54.8 & 43.2 & 24.9 & 50.7 & 59.5 \\
\hline $23 a$ & 54.6 & 19.1 & 35.0 & 7.0 & 5.0 & $65 \%$ & 55.9 & 55.3 & 46.7 & 35.8 & 53.2 & 59.5 \\
\hline $23 b$ & 35.0 & 9.3 & 39.0 & 3.0 & 13.0 & $60 \%$ & 68.2 & 67.0 & 50.3 & 36.4 & 62.7 & 50.7 \\
\hline 24 & 25.1 & 4.2 & 36.0 & 3.0 & 12.0 & $60 \%$ & 58.8 & 50.4 & 37.1 & 34.0 & 43.2 & 46.8 \\
\hline 25 & 33.7 & 5.8 & 40.0 & 8.0 & 5.0 & $65 \%$ & 63.4 & 61.9 & 50.3 & 41.1 & 57.5 & 72.0 \\
\hline $26 a$ & 101.9 & 29.8 & 40.0 & 8.0 & 5.0 & $60 \%$ & 70.7 & 69.4 & 54.6 & 29.6 & 66.6 & 72.0 \\
\hline $26 b$ & 132.6 & 64.8 & 35.0 & 7.0 & 5.0 & $65 \%$ & 55.5 & 54.7 & 51.6 & 38.4 & 53.8 & 59.5 \\
\hline 27 & 71.6 & 17.1 & 40.0 & 8.0 & 5.0 & $60 \%$ & 71.7 & 70.2 & 48.4 & 25.1 & 65.0 & 72.0 \\
\hline 28 & 249.4 & 191.8 & $42.0^{*}$ & 3.0 & 14.0 & $60 \%$ & 70.2 & 67.9 & 41.4 & 34.3 & 58.2 & 54.6 \\
\hline 29 & 149.0 & 63.1 & $12.0^{*}$ & 4.0 & 3.0 & $60 \%$ & 21.2 & 20.5 & 15.8 & 10.5 & 18.9 & 69.9 \\
\hline 30 & 31.8 & 11.6 & 35.0 & 7.0 & 5.0 & $65 \%$ & 53.9 & 52.1 & 43.8 & 35.7 & 46.9 & 59.5 \\
\hline 31 & 78.8 & 27.8 & 40.0 & 8.0 & 5.0 & $65 \%$ & 61.9 & 59.6 & 49.2 & 42.4 & 55.5 & 72.0 \\
\hline
\end{tabular}

*ID 28: 35 Gy á $2.5 \mathrm{~Gy}$ with simultaneous integrated boost on the adrenal lesion to a cumulative dose of $42 \mathrm{~Gy}$ á $3 \mathrm{~Gy}$ *ID 29: 45 Gy á $1.8 \mathrm{~Gy}$ with sequential boost on the adrenal lesion of $12 \mathrm{~Gy}$ á $4 \mathrm{~Gy}$

PTV planning target volume, GTV gross tumor volume, TD total dose, SD single dose

related to the adrenal lesions, including back pain, flank pain, and abdominal pain; the applied RT had a positive effect on the pain situation in both patients. In 44\% (15/ 34) adrenal lesions occurred synchronously during the diagnosis of the primary tumor, while $56 \%(19 / 34)$ of the metastases were diagnosed metachronously ( $>3$ months after initial diagnosis). The median time between the diagnosis of the primary tumor and the occurrence of adrenal metastases was 12.3 months (range: $0-169.0$ months). Of all, $19(19 / 31,61 \%)$ patients received systemic tumor therapy, such as chemo, immuno- or hormone therapy, within four weeks before or after RT. It was ensured that this therapy was administered only in exceptional cases at the same time. The median planning target volume (PTV) was $76.8 \mathrm{ml}$ (range: 24.1-434.8). Further planning and irradiation parameters are shown in Table 2 . 


\section{Outcome}

Median follow-up for all patients was 9.8 months (range: 0-120.5), and 14.4 months (range: 1.4-120.5) for patients alive at the time of analysis. The mean LC was 79.0 months (95\%-CI: 53.6-104.3; the median was not reached). We used competing-risk-analysis to estimate the probability of local relapse in our cohort. The 1-year chance of developing a local tumor progression was 25.9\%, see also Table 3 and Fig. 1c. Median OS was 18.7 months (CI: 8.1-29.2; Fig. 1a), 64\% of the patients were alive one year after RT. Median PFS was 2.3 months (CI: 1.0-3.6; Fig. 1b). After one year, 19\% of the patients did not experience a distant failure (see also Table 3). We divided the patients into two groups according to PTV, with a threshold value of $80 \mathrm{ml}$ and adjusted for dose using Cox regression (Fig. 1d). A PTV $<80 \mathrm{ml}$ significantly influences LC $(p=0.033)$. For $\mathrm{BED}_{10}$, the $\mathrm{ROC}$ analysis could not identify a specific threshold; hence, we tested it as a continuous prognostic variable. No significant impact on LC $(p=0.115)$ could be found.

Figure 2 shows an example case of a successfully SBRT-treated patient whos adrenal gland metastasis was no longer detectable after SBRT.

\section{Treatment toxicity}

SBRT of the adrenal metastases was overall very well tolerated. No acute or late therapy-associated side-effects > grade 2 occurred. The most common side-effects included mild nausea, fatigue, loss of appetite, and abdominal pain, see Table 4. All acute symptoms were well treatable and were already decreasing after a short time. Two patients developed a mild adrenal insufficiency after SBRT and had to be treated with hormone substitution. Both patients were each irradiated with a cumulative dose of $35 \mathrm{~Gy}$ and $25 \mathrm{~Gy}$ with a single dose of $7 \mathrm{~Gy}$ and 5 Gy prescribed to the 65 and $60 \%$ isodose, respectively (ID 4 and 23, depicted in Table 2). No patient developed gastrointestinal ulcers, stopped SBRT early, or died during treatment.

\section{Discussion}

Hypofractionated low-dose stereotactic body radiation therapy of adrenal metastases has become increasingly important in recent years. We analyzed a cohort of 31 patients with 34 irradiated adrenal lesions. Occurred side-effects were extremely rare, mild, and well treatable. Local control was promising and comparable to other studies [6, 13, 14, 22-25].

In the past, surgical resection of adrenal metastases (adrenalectomy) was the gold standard for patients with isolated adrenal gland metastases. Especially if the metastases were isolated, the open or laparoscopic adrenalectomy was and is the first choice, depending on the tumor size and patient condition. Certainly, the postoperative side-effects associated with this invasive treatment should not be underestimated. Since 1992, the laparoscopic approach has been increasingly used as the postoperative complications are significantly lower, and both the duration and intensity, as well as the hospital stay can be reduced [3, 26-28].

Other treatment options include ablative procedures, such as cryoablation or radiofrequency ablation. However, these ablative measures bear a higher risk of blood pressure derailment and intensive post-intervention care [29]. In contrast to these invasive methods, SBRT is a non-invasive alternative with an outstanding risk-benefit profile. Due to the comparatively gentle tumor tissue inactivation and the biological effects, there is no associated secretion of catecholamines during therapy; thus, a therapy-associated hypertensive crisis is avoided, and adrenal hormonal function can be preserved in most cases, as our results showed [30].

According to Toesca et al., who compared the glomerular filtration rate (GFR) before and after stereotactic

Table $3 \mathrm{LF}, \mathrm{PFS}$, and OS in total and depending on time

\begin{tabular}{|c|c|c|c|c|c|}
\hline & & & LF & PFS & OS \\
\hline \multicolumn{3}{|c|}{ Event (progress/death) - absolute/(\%) } & $7(20.6)$ & $25(80.6)$ & $17(54.8)$ \\
\hline \multicolumn{3}{|c|}{ No event - absolute/(\%) } & $27(79.4)$ & $6(19.4)$ & $14(45.2)$ \\
\hline \multicolumn{3}{|c|}{ Time (in months) } & mean: 79.0 & median: 2.3 & median: 18.7 \\
\hline \multicolumn{3}{|c|}{ 95\%-Cl (in months) } & $53.6-104.3$ & $1.0-3.6$ & $8.1-29.2$ \\
\hline & \multicolumn{4}{|c|}{ Proportion surviving after } & \\
\hline & 6-months & 12-months & 18-months & 24-months & \\
\hline OS & $80 \%$ & $64 \%$ & $50 \%$ & $34 \%$ & \\
\hline \multirow[t]{3}{*}{ PFS } & $30 \%$ & $19 \%$ & $15 \%$ & $15 \%$ & \\
\hline & \multicolumn{4}{|c|}{ Probability of LF after } & \\
\hline & 6-months & 12-months & 18-months & 24-months & \\
\hline LF & $6 \%$ & $26 \%$ & $26 \%$ & $26 \%$ & \\
\hline
\end{tabular}

LF local failure, PFS progression-free survival, OS overall survival, $\mathrm{Cl}$ confidence interval 


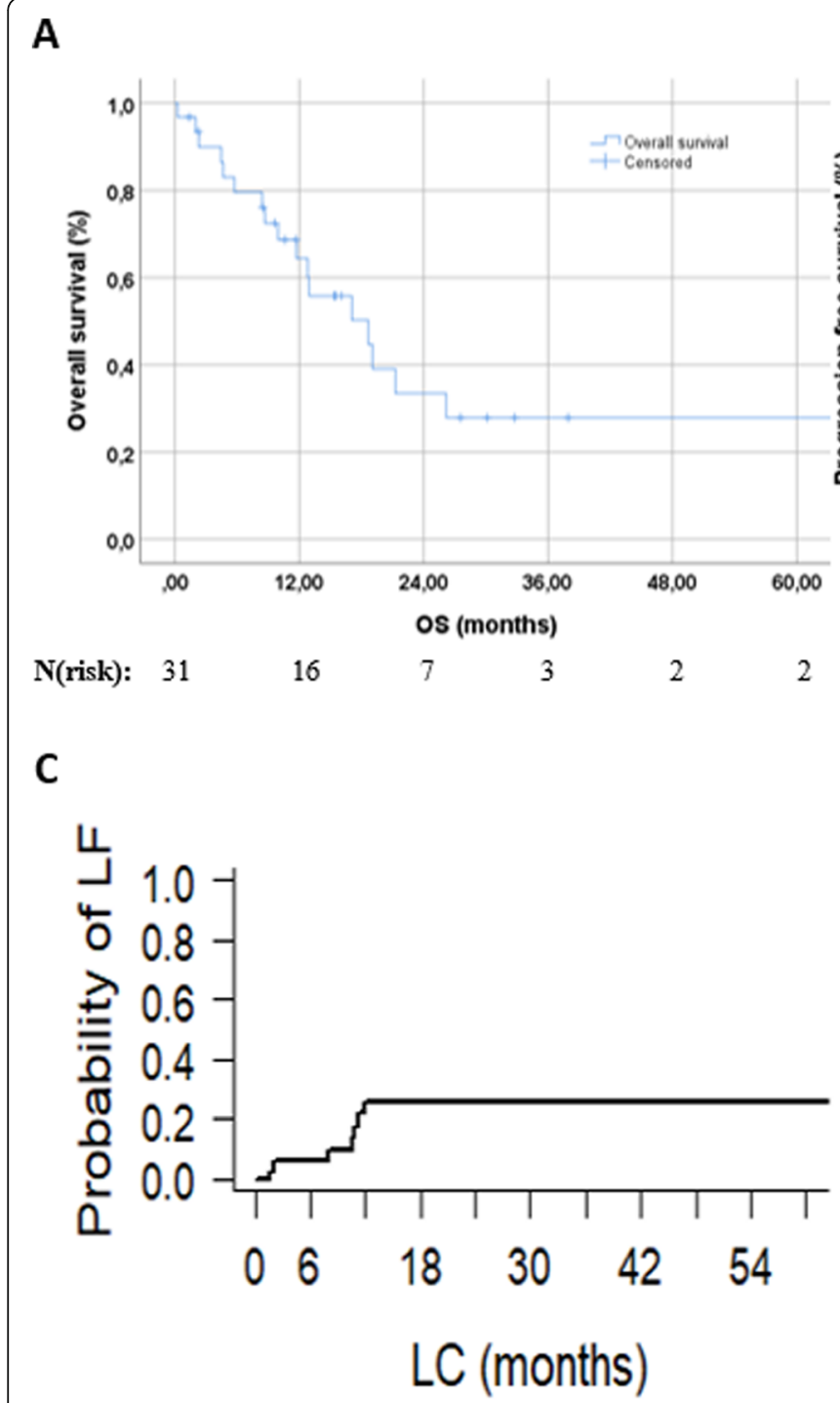

B

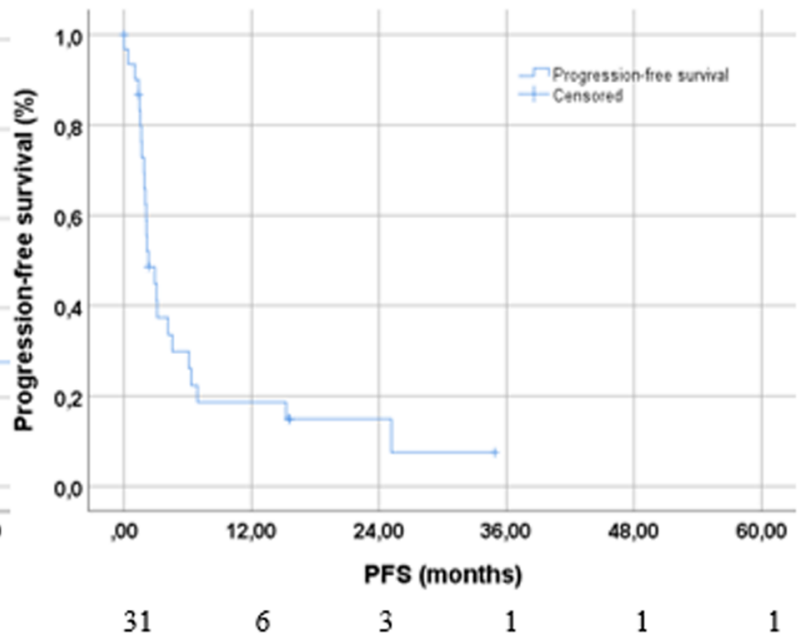

D

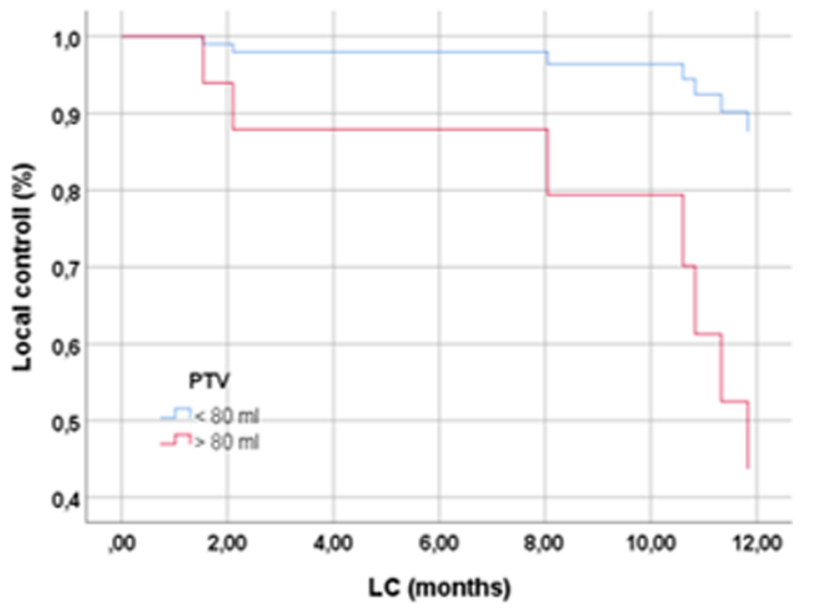

N(risk): $\quad \begin{array}{lllllll}34 & 11 & 7 & 2 & 2 & 2\end{array}$

Fig. 1 a OS of patients with AGMs treated with low-dose SBRT; b PFS of treated patients; c Probability of local failure; $\mathbf{d} L C$ divided into PTV </> $80 \mathrm{ml}$ and adjusted for dose $(p=0.033)$

treatment, kidney function is also little or not affected by SBRT. According to several studies conducted in the past, LC after SBRT is equaled to or even better than ablative procedures [13].

The studies investigating RT of adrenal gland metastases conducted in the last six years report a 1-year LC rate between 73 - 97\% [6, 13, 14, 22-25]. Our 1-year probability of local failure was $25.9 \%$. The effect of $\mathrm{BED}_{10}$ on local tumor control has already been investigated in several studies, and it was noted that a higher applied dose tends to be associated with a better local outcome. Ippolito et al. reported an LC-rate of $>70 \%$ if $\mathrm{BED}_{10}$ is $>60 \mathrm{~Gy}$ and $>90 \%$ with $\mathrm{BED}_{10}>90 \mathrm{~Gy}$. In the cohort of Chance et al. appeared no local relapses by applying a $\mathrm{BED}_{10}>100 \mathrm{~Gy}$ and Rudra et al. noted that all occurred local failures in their cohort were treated with the lowest mean $\mathrm{BED}_{10}$ of $43.2 \mathrm{~Gy}[8,14,31]$. In our patients, we could not find any significant influence of $\mathrm{BED}_{10}$ on local control. Only a non-significant trend towards better local control with higher BED could be identified $(p=0.115)$. All adrenal metastases that showed a local relapse after RT were treated with a $\mathrm{BED}_{10}$ of 37.5 and $59.5 \mathrm{~Gy}$. No local failure occurred with $\mathrm{BED}_{10}>$ $59.5 \mathrm{~Gy}$. In order to better assess the correlation between $\mathrm{BED}_{10}$ and local tumor control in adrenal metastases, a larger group of patients would be necessary.

Therapy-associated toxicity is an important limiting factor of any new therapy. SBRT proved to be 

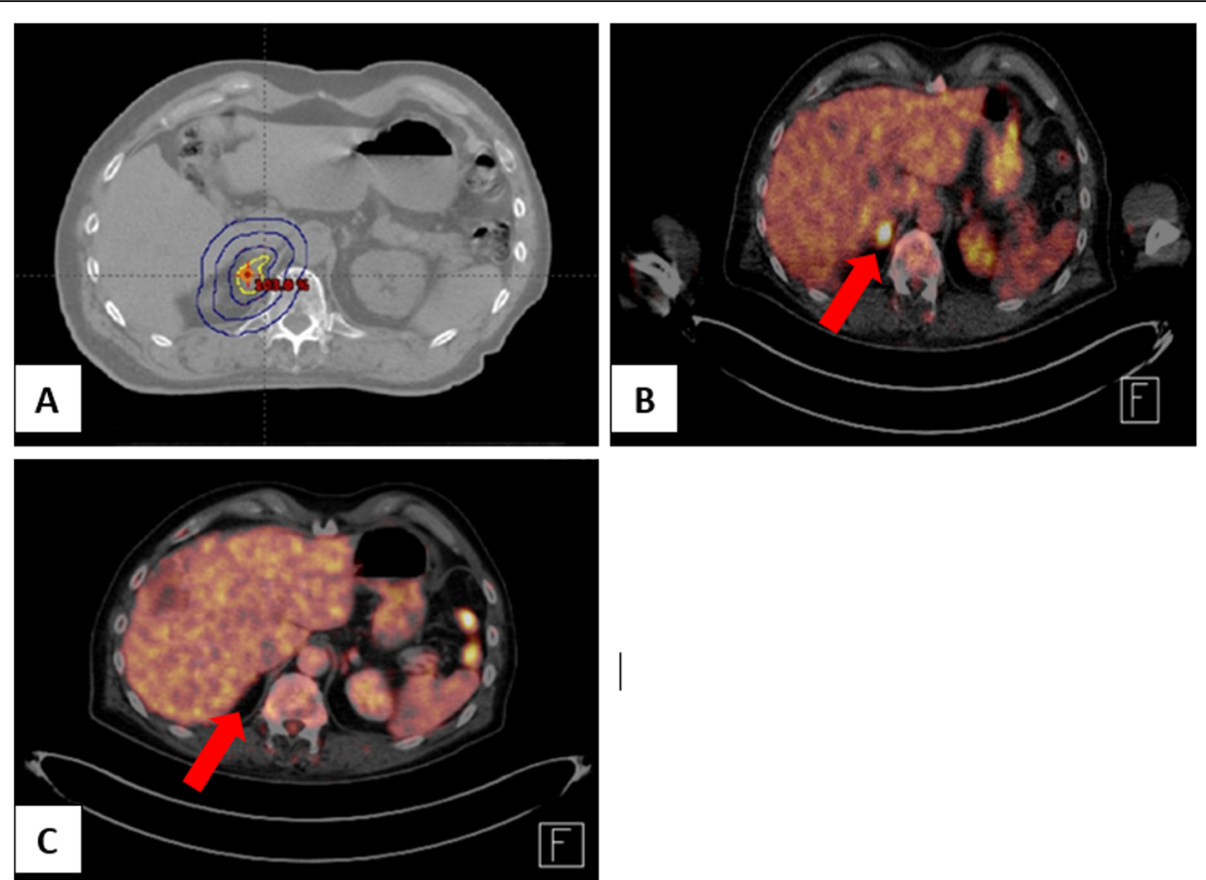

Fig. 2 Example of a patient with malignant melanoma, who successfully underwent SBRT of adrenal gland metastasis. a CT scan and treatment plan before RT with 40 Gy in 5 fractions. b PET scan of the adrenal lesion before treatment (marked with red arrow). c PET scan four weeks after SBRT

beneficial in our study as no side-effects >grade 2 occurred. Also, in other studies on SBRT of adrenal metastases, no grade $3-5$ toxicity was reported $[8$, $14,24,25,32,33]$, except for grade 3 diarrhea in the patient cohort of Zhao et al. [6]. Due to the high precision and exact application of radiation, both acute and late toxicities are rare and usually very mild. However, due to the respiratory dependence of adrenal tumor volume, dosimetry may often prove very difficult for the multiple adjacent organs at risk,

Table 4 Acute and late toxicities after SBRT of adrenal gland metastases

\begin{tabular}{lll}
\hline Acute toxicity $(\boldsymbol{n}=\mathbf{3 1})$ & Grade $\mathbf{1}$ absolute/ (\%) & Grade $\mathbf{2}$ absolute/ (\%) \\
Nausea & $2(6.5)$ & $4(12.9)$ \\
Vomiting & $0(0)$ & $1(3.2)$ \\
Abdominal pain & $2(6.5)$ & $2(6.5)$ \\
Loss of weight & $1(3.2)$ & $1(3.2)$ \\
Loss of appetite & $2(6.5)$ & $1(3.2)$ \\
Diarrhea & $2(6.5)$ & $0(0)$ \\
Constipation & $1(3.2)$ & $1(3.2)$ \\
Fatigue & $6(19.4)$ & $5(16.1)$ \\
Throbbing pain & $0(0)$ & $2(6.5)$ \\
Adrenal insufficiency & $0(0)$ & $2(6.5)$ \\
Radiogenic gastritis & $0(0)$ & $1(3.2)$ \\
Flatulence & $1(3.2)$ & $1(3.2)$ \\
Late toxicity $(\boldsymbol{n}=\mathbf{3 1 )}$ & Grade $\mathbf{1}$ absolute/ (\%) & Grade 2 absolute/ (\%) \\
Gastrointestinal & $4(12.9)$ & $0(0)$ \\
Fatigue & $1(3.2)$ & $3(9.7)$ \\
Headache & $0(0)$ & $2(6.5)$ \\
Loss of weight & $0(0)$ & $1(3.2)$
\end{tabular}


such as the liver and bowel. Excellent and precise respiratory tumor motion management is crucial during the radiation treatment, and the dose must be reduced or adjusted to the circumstances, especially to avoid late toxicity [24].

Furthermore, according to Holy et al., treatment on an empty stomach has a positive effect on the described side-effects, as it ensures a better reproducibility of the position of the internal organs, and the intestinal movements can be minimized [32]. Another factor influencing the onset of late-toxicities is the tumor volume. The smaller the irradiated lesion, the less likely it is the appearance of late therapy-associated side-effects, as Zhao et al. found in their study of 2018 [6]. Overall, our LC and treatment-associated toxicity results are very similar to previous studies, supporting the assumption that SBRT is a valid treatment option for metastases of the adrenal gland.

Limitations of the study were the relatively small patient cohort, the short follow-up, and the retrospective design. The heterogeneously designed irradiation regimens were also among the limiting factors, as no consistent treatment guidelines regarding fractionation and dose exist.

\section{Conclusion}

Low-dose SBRT is an excellent, effective, and safe method to treat metastases in the adrenal gland. Due to the exact application and the precise dosing of the radiation, all toxicities have been low and easy to treat. High-precision RT is a promising alternative for patients for whom surgical treatment is not possible due to poor general conditions or other treatment-limiting factors. Based on our results, which support existing studies, SBRT should be considered as a therapy option for adrenal metastases as an alternative to surgical or systemic treatment. Prospective studies are nevertheless necessary for the validation of these results.

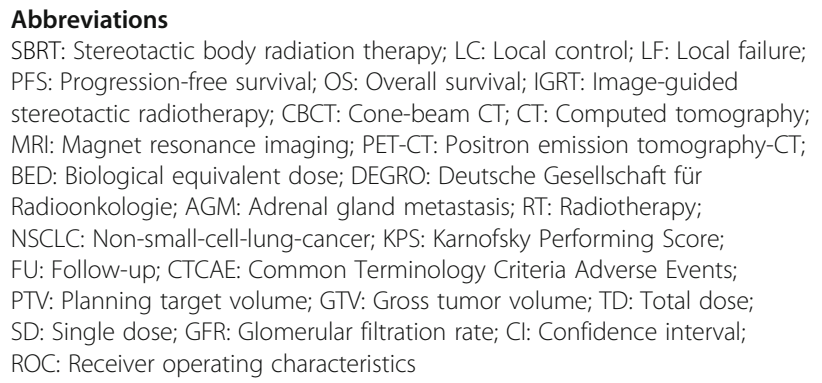

Abbreviations

SBRT: Stereotactic body radiation therapy; LC: Local control; LF: Local failure: PFS: Progression-free survival; OS: Overall survival; IGRT: Image-guided stereotactic radiotherapy; CBCT: Cone-beam CT; CT: Computed tomography; MRI: Magnet resonance imaging; PET-CT: Positron emission tomography-CT; BED: Biological equivalent dose; DEGRO: Deutsche Gesellschaft für Radioonkologie; AGM: Adrenal gland metastasis; RT: Radiotherapy; NSCLC: Non-small-cell-lung-cancer; KPS: Karnofsky Performing Score; FU: Follow-up; CTCAE: Common Terminology Criteria Adverse Events; PTV: Planning target volume; GTV: Gross tumor volume; TD: Total dose; SD: Single dose; GFR: Glomerular filtration rate; Cl: Confidence interval; ROC: Receiver operating characteristics

\section{Acknowledgements}

Not applicable.

\section{Consent of publication}

This manuscript does not contain any images and information that endanger the anonymity of a patient. Thus, no other form of informed consent is required. The use of patient data for any further analysis was approved and confirmed by the local ethics committee.

\section{Authors' contributions}

TV and KAK collected and analyzed the data and planned and designed the experiments. TV wrote the paper using the expert urological and radiotherapeutic advice about the adrenal gland and metastases found here provided by JEG and MMEV. MMEV also helped with the statistical analysis of the data. Analyzing of treatment planning and all physical data was supervised by MO. SEC supervised the project. All authors revised the paper, read and approved the final version of the manuscript.

\section{Funding}

Not applicable.

Availability of data and materials

All datasets used to create and support the results and conclusions of this article can be found within the article.

\section{Ethics approval and consent to participate}

Analysis of patient records and data collection took place after being approved by the local ethics committee of the Technical University Munich (TUM), vote number 307/19.

The ethics vote for this retrospective analysis is available in writing. No other form of explicit informed consent was necessary.

\section{Competing interests}

The authors declare that they have no competing interests.

\section{Author details}

${ }^{1}$ Department of Radiation Oncology, Technical University of Munich (TUM), Ismaninger Straße 22, Munich, Germany. ${ }^{2}$ Institute of Radiation Medicine (IRM), Helmholtz Zentrum München, Ingolstädter Landstraße 1, Neuherberg, Germany. ${ }^{3}$ Deutsches Konsortium für Translationale Krebsforschung (DKTK), Partner Site Munich, Heidelberg, Germany. ${ }^{4}$ Department of Urology, Technical University of Munich (TUM), Ismaninger Straße 22, Munich, Germany.

Received: 19 December 2019 Accepted: 2 June 2020

Published online: 08 June 2020

\section{References}

1. Kung AW, Pun KK, Lam K, et al. Addisonian crisis as presenting feature in malignancies. Cancer. 1990;65:177-9.

2. Ilias I, Pacak K. Diagnosis and Management of Tumors of the adrenal medulla. Horm Metab Res. 2005:37(12):717-21.

3. Uberoi J, Munver R. Surgical Management of Metastases to the adrenal gland: open, laparoscopic, and ablative approaches. Curr Urol Rep. 2009;10: $67-72$.

4. Lam KY, Lo CY. Metastatic tumours of the adrenal glands: a 30-year experience in a teaching hospital. Clin Endocrinol. 2002;56:95-101.

5. Abrams HL, Spiro R, Goldstein N. Metastases in carcinoma: analysis of 1000 autopsied cases. Cancer. 1950:3:74-85.

6. Zhao X, Zhu X, Fei J, et al. Short-term outcomes and clinical efficacy of stereotactic body radiation therapy (SBRT) in treatment of adrenal gland metastases from lung cancer. Rad Oncol. 2018;13(1):205.

7. Soffen EM, Solin $\amalg$, Rubenstein JH, et al. Palliative Radiotherapy for Symptomatic Adrenal Metastases. Cancer. 1990;65:1318-20.

8. Ippolito E, D'Angelillo RM, Fiore M, et al. SBRT: a viable option for treating adrenal gland metastases. Rep Pract Oncol Radiother. 2015;20:484-90.

9. Welch BT, Callstrom MR, Carpenter PC, et al. A single-institution experience in image-guided thermal ablation of adrenal gland metastases. J Vasc Interv Radiol. 2014;25:593-8.

10. Botsa El, Thanou IL, Papatheodoropoulou AT, et al. Thermal ablation in the Management of Adrenal Metastasis Originating from non-small cell lung Cancer: a 5-year single-center experience. Chin Med J. 2017;130(17):2027-32.

11. Hasegawa T, Yamakado K, Nakatsuka A, et al. Unresectable adrenal metastases: clinical outcomes of radiofrequency ablation. Radiology. 2015; 277:584-93. 
12. Gao XL, Zhang KW, Tang MB, et al. Pooled analysis for surgical treatment for isolated adrenal metastasis and non-small cell lung cancer. Interact Cardiovasc Thorac Surg. 2017;24:1-7.

13. Toesca DAS, Koong AJ, von Eyben $R$, et al. Stereotactic body radiation therapy for adrenal gland metastases: outcomes and toxicity. Adv Rad Oncol. 2018;3:621-9.

14. Rudra S, Malik R, Ranck MC, et al. Stereotactic body radiation therapy for curative treatment of adrenal metastases. Technol Cancer Res Treat. 2013;12: 217-24

15. Palma DA, Louie AV, Rodrigues GB. New strategies in stereotactic radiotherapy for Oligometastases. Clin Cancer Res. 2015;21(23):198-204.

16. Salama JK, Hasselle MD, Chmura SJ, et al. Stereotactic body radiotherapy for multisite extracranial oligometastases. Cancer. 2012;118:2962-70.

17. Riegel AC, Kara Bucci M, Mawlawi OR, et al. Defining internal target volume using positron emission tomography for radiation therapy planning of moving lung tumors. J Appl Clin Med Phys. 2014;15(1):279-89.

18. Sindoni A, Minutoli F, Pontoriero A, et al. Usefulness of four dimensional (4D) PET/CT imaging in the evaluation of thoracic lesions and in radiotherapy planning: review of the literature. Lung Cancer. 2016;96:78-86.

19. Scrucca L, Santucci A, Aversa F. Competing risk analysis using R: an easy guide for clinicians. Bone Marrow Transplant. 2007;40(4):381-7.

20. Dutz A, Lock S. Competing risks in survival data analysis. Radiother Oncol. 2019;130:185-9.

21. Park C, Papiez $L$, Zhang $S$, et al. Universal survival curve and single fraction equivalent dose: useful tools in understanding potency of ablative radiotherapy. Int J Radiat Oncol Biol Phys. 2008;70(3):847-52.

22. Franzese C, Franceschini D, Cozzi L, et al. Minimally invasive Stereotactical Radio-ablation of Adrenal Metastases as an alternative to surgery. Cancer Res Treat. 2017:49:20-8.

23. Ahmed KA, Barney BM, Macdonald K, et al. Stereotactic body radiotherapy in the treatment of adrenal metastases. Am J Clin Oncol. 2013;36:509-13.

24. Plichta $\mathrm{K}$, Camden N, Furqan M, et al. SBRT to adrenal metastases provides high local control with minimal toxicity. Adv Rad Oncol. 2017;2:581-7.

25. König L, Hafner MF, Katayama S, et al. Stereotactic body radiotherapy (SBRT) for adrenal metastases of oligometastatic or oligoprogressive tumor patients. Radiat Oncol. 2020;15(1):30.

26. Hauch A, Al-Qurayshi Z, Kandil E. Factors associated with higher risk of complications after adrenal surgery. Ann Surg Oncol. 2015;22:103-10.

27. Prinz RA. A comparison of laparoscopic and open adrenalectomies. Arch Surg. 1995;130:489-92.

28. Smith CD, Weber C, Amerson RJ. Laparoscopic Adrenalectomy: new gold standard. World J Surg. 1999;23:389-96

29. Men M, Ye X, Fan W, et al. Short-term outcomes and safety of computed tomography-guided percutaneous microwave ablation of solitary adrenal metastasis from lung Cancer: a multi-center retrospective study. Korean J Radiol. 2016;17:864-73.

30. Eldaya RW, Paulino AC, Blanco Al, et al. Preservation of adrenal function after successful stereotactic body radiation therapy of metastatic renal cell carcinoma involving the remaining contralateral adrenal gland. Pract Rad Oncol. 2012:2:270-3.

31. Chance WW, Nguyen QN, Mehran R, et al. Stereotactic ablative radiotherapy for adrenal gland metastases: Factors influencing outcomes, patterns of failure, and dosimetric thresholds for toxicity. Pract Radiat Oncol. 2017;7(3): e195-203.

32. Holy R, Piroth M, Pinkawa M, et al. Stereotactic body radiation therapy (SBRT) for treatment of adrenal gland metastases from non-small cell lung Cancer. Strahlenther Onkol. 2011;187:245-51.

33. Shah MM, Isrow D, Fareed MM, et al. Single institution experience treating adrenal metastases with stereotactic body radiation therapy. J Can Res Ther 2019;15:27-32.

\section{Publisher's Note}

Springer Nature remains neutral with regard to jurisdictional claims in published maps and institutional affiliations.

Ready to submit your research? Choose BMC and benefit from:

- fast, convenient online submission

- thorough peer review by experienced researchers in your field

- rapid publication on acceptance

- support for research data, including large and complex data types

- gold Open Access which fosters wider collaboration and increased citations

- maximum visibility for your research: over $100 \mathrm{M}$ website views per year

At $\mathrm{BMC}$, research is always in progress.

Learn more biomedcentral.com/submissions 